\title{
The infant lung function model: a mechanical analogue to test infant lung function equipment
}

\author{
U. Frey*, B. Reinmann*, J. Stocks ${ }^{\#}$
}

\begin{abstract}
The infant lung function model: a mechanical analogue to test infant lung function equipment. U. Frey, B. Reinmann, J. Stocks. C) ERS Journals Ltd 2001.

ABSTRACT: To facilitate international multicentre studies and quality control of infant pulmonary function measurements, the European Respiratory Society-American Thoracic Society (ERS-ATS) working group for infant lung function testing aims to develop specifications for standardized infant lung function equipment and software. However, a standardized test device is also needed to test whether existing infant lung function equipment is able to meet these requirements.

The authors have built a "mechanical model baby" consisting of a linear pump which can reproduce prerecorded tidal flow waveforms with a precision of $0.5 \%$ (full stroke), enabling the simulation of tidal and forced flow patterns. This linear pump can be connected to a series of copper lung volumes (range 50-300 mL) with known time constants, so that lung volumes can be reproduced with a precision of $\pm 1 \%$ at frequencies $10-120 \mathrm{bpm}$. Five airflow resistors were built using sinter material.

When assessed using flows $0-300 \mathrm{~mL} \cdot \mathrm{s}^{-1}$ all resistors showed a quasilinear pressure/ flow relationship, with slopes $1.0-5.6 \mathrm{kPa} \cdot \mathrm{L}^{-1} \cdot \mathrm{s}$. These resistances could be reproduced with a precision of $\pm \mathbf{2 . 5} \%$. The infant lung model can also be used to assess frequency responses of infant lung function equipment, since the pump is capable of delivering low amplitude volumes up to $20 \mathrm{~Hz}$ in a pseudorandom noise manner.

In summary, based on error estimations, this infant lung model is able to test whether or not infant lung function equipment meets the requirements suggested by the European Respiratory Society-American Thoracic Society standardization group, that is: flow measurements within $\pm \mathbf{2 . 5 \%}$, volume and resistance measurements within $\pm 5 \%$, frequency response: magnitude attenuation $<\mathbf{\pm 1 0} \%$ and phase shift $< \pm 3$ degrees at $10 \mathrm{~Hz}$. Eur Respir J 2001; 17: 755-764.
\end{abstract}

*University Hospital of Berne, Inselspital, Berne, Switzerland, 3010 and \#Portex Anaesthesia, Intensive Care and Respiratory Medicine Unit, Institute of Child Health, London, UK.

Correspondence: U. Frey, Dept of Paediatrics, University Hospital of Berne, Inselspital, Berne, Switzerland Fax: 41316329484

Keywords: Equipment and supplies infant

model

plethysmography

respiratory function tests

Received: September 81999

Accepted after revision December 29 2000

This work has been supported in part by Erich Jaeger GmbH, Hoechberg, Germany.
Lung function measurements in the first year of life have had an increasing impact on the understanding of normal lung development and on the early detection and monitoring of lung disease. Several lung function techniques have been proposed to access the highly complex mechanical properties of the infant lung [1]. In order to assess infant lung mechanics, it is necessary to measure resistance, lung volumes and both tidal and forced flow patterns with a high degree of accuracy. Assessment of lung mechanics in infants requires higher technical demands of the equipment than when studying adults, because of a smaller signal to noise ratio and higher respiratory frequency. Furthermore, calibration of equipment is often performed under highly specialized conditions, while the overall performance of the equipment as an integrated whole is rarely assessed. Hand held calibration syringes do not allow maintenance of a constant pumping frequency and the air within them is subject to warming by the investigator's hand. Consequently, the thermal behaviour of these syringes is both unknown and highly variable.

It is recognized that automated calibration and equipment testing procedures are potentially superior to manual approaches, and some manufacturers now provide semiautomatic calibration and testing devices for their infant lung function devices. However, most of these have limited capabilities. Since it is impossible to check infant lung function equipment in the factory using infant "test subjects", most equipment for this age range is released without under going any in vivo validation. The lack of an appropriate lung model to assess complex parameters such as plethysmographic lung volumes, resistance and forced flows has meant that even in vitro assessments have been minimal, these being largely left to the initiative of individual investigators after release of the equipment. This lack of properly standardized devices and protocols for assessing infant lung function equipment has made it difficult to compare results obtained with different systems or from different centres. This also makes quality control of pulmonary function labs difficult and obstructs the development of new equipment and software.

To facilitate international multicentre studies and improve quality control of infant pulmonary function measurements, the European Respiratory Society-American Thoracic Society (ERS-ATS) working group for infant lung function testing has recently developed 
specifications for standardized infant lung function equipment and software [2, 3]. However, to test whether existing infant lung function equipment is able to meet these requirements, a standardized test device is also needed. Such a test device should be able to simulate pressure, flow and volume changes in the order of magnitude that occur during infant lung function tests.

The aim of this project was to design and describe the physical properties of a "breathing mechanical model baby", in other words a mechanical infant lung function model which could reliably simulate infant flow patterns, airway resistance and lung volumes. Such a device would be capable of testing not only the performance of individual components, but the overall performance of the equipment. Furthermore it was aimed to measure the precision and repeatability of pressures and flows generated by this model under various conditions.

For clarity, the protocol is presented in four sections, each presenting the rationale, methods and the results of a particular aspect of the infant lung model. Firstly, the dimensions and physical properties of the infant lung model pump, including frequency range and accuracy of its tidal volume and flow output are defined. This includes the ability of the model pump to reproduce prerecorded flows. Secondly, the mechanical and thermal properties of the model volumes representing infant functional residual capacities are described. Thirdly, the flow properties of various flow resistor elements representing infant airway resistance are presented. Finally the frequency response of the lung model up to $10 \mathrm{~Hz}$ is described $[2,3]$.

\section{Methods and results}

\section{Study design}

An infant lung model has to be capable of simulating flows, volumes, resistances and pressures as occurring in $2-12 \mathrm{~kg}$ infants, with a precision that meets the required accuracy criteria for infant lung function measurements defined in the ERS-ATS standards [2, 3]. In this study, the simplest possible solution of a mechanical analogue of infant breathing was designed. This single compartment infant model consists of a linear pump and serial volume and resistance elements. The composition of the infant lung model varied depending on whether lung volumes, flows or airway resistances were to be simulated. The infant lung model set-up is shown in detail in figure 1 . The internal diameter of the copper volume elements was $51.4 \mathrm{~mm}$ and length varied from $10.1 \mathrm{~mm}$ for the $50 \mathrm{~mL}$ volume (V50) to $130.8 \mathrm{~mm}$ for the $300 \mathrm{~mL}$ volume (V300) [4].

\section{Experimental protocol}

Tidal flow and volume output of the linear model pump. Rationale. The ideal infant lung model pump needs to meet high technical standards and to fulfil at least six criteria: 1) It should be small enough to fit into an infant plethysmograph [5]. 2) It should not vibrate and thus not cause signal to noise prob- lems. Vibrations are particularly disturbing if their frequency is $<10 \mathrm{~Hz}$, since these will usually be picked up by the infant lung function pressure transducers. The frequency of such vibrations are very unpredictable since they depend on the resonance behaviour of the infant lung function equipment. Similarly, difficulties are introduced by pumps that develop heat or strong magnetic fields, which can introduce signal drifts. 3) It must be able to deliver small tidal volumes accurately. 4) It should be fast enough to enable rapid changes in flow such as occur in infants. 5) Ideally, it should be able to reproduce prerecorded infant tidal volume patterns, since infant lung function equipment is often not only used to measure lung volume or airflow resistance, but to determine indices from tidal or forced flow and volume waveforms (such as breathing frequency, inspiratory and expiratory time, tidal volume, time to peak expiratory flow and maximal flow at fixed volume intervals. During regular sinusoidal pumping, such distinct points of the tidal flow waveform are easy to determine and triggering mechanisms rarely fail. However, during irregular breathing, the situation becomes much more complex. An infant lung model should therefore have the ability to reproduce flow wave forms as they occur in real infants. 6) Finally, an infant lung model pump should be able to produce low amplitude sinusoidal volume changes up to $10 \mathrm{~Hz}$, so that the frequency response of infant lung function equipment can be tested.

Methods and results. The infant model (fig. 1) consisted of a computer-driven linear electromagnetic motor with two different exchangeable pistons (Scireq Inc., Montreal, Canada). The linear motor itself did not cause significant warming or mechanical vibration $[4,5]$.

The linear pump has been described and validated previously [6]. Briefly, dependent on the piston used (small or large), the pump was able to deliver sinusoidal or prerecorded tidal volumes over a range of 0.25 $4.5 \mathrm{~mL}$ (small) and 10-100 $\mathrm{mL}$ (large). These tidal stroke volumes of the piston $(V \mathrm{p})$ were calculated from the physical movement of the piston by the pump's

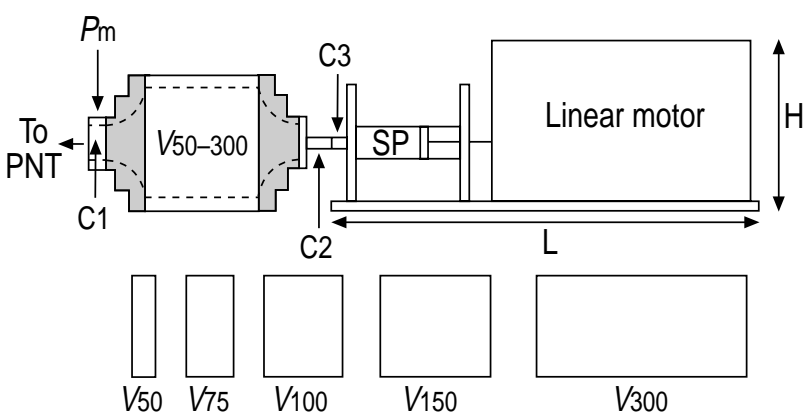

Fig. 1. - Diagram of the mechanical infant lung model. This model consists of a computer driven linear pump attached to various exchangeable lung volume elements $\left(V_{50-300}\right)$ that can be inserted into the model as shown. $\mathrm{C} 1-3$ : Connectors 1-3; PNT: pneumotachograph; $\square$ : copper adaptor; L: Length $=545 \mathrm{~mm} ; \mathrm{H}$ : Height $=120 \mathrm{~mm}$, Width $=305 \mathrm{~mm}$. The model pressure $P \mathrm{~m}$ represents the airway opening pressure $(P$ ao $)$ in the real test situation. 
driving software, and output as an accurate digital signal, which was used as a reference signal. Low tidal volumes (for frequency response measurements) can be delivered up to a frequency of $20 \mathrm{~Hz}$. The accuracy of the sinusoidal tidal volume output has been described previously [6] and ranges within $0.5 \%$ of full stroke. However, if the delivered stroke volume is only $10 \%$ of the full stroke volume, the precision decreases proportionally and has been found to be up to $5 \%$ (unpublished data). Thus the piston of the pump must be chosen so that the full stroke volume is of the same order of magnitude as the tidal volume required for the tests. In this infant lung model setup, the minimal absolute error would be $0.023 \mathrm{~mL}$ and $0.5 \mathrm{~mL}$, respectively, when using the small and large pistons. The standards for infant lung function equipment state that the smallest absolute error of flow measurements should be $< \pm 2.5 \%$ or $1 \mathrm{~mL}$ [3]. Thus, these standards are fulfilled by the current model pump.

One of the big advantages of using a computer driven linear pump is its ability to reproduce prerecorded flow patterns. Thus the model pump should be able to simulate flow patterns of tidal breathing or forced expiratory manoeuvres. The authors did not determine the pump's ability to reproduce flow signals in detail since this has been previously described [6]. However, as an example, the flow pattern was simulated over a 16-s period from a 6 month old infant, using a sampling rate of $200 \mathrm{~Hz}$ (accuracy 12 Bit; Rasp Analysis Software, Physiologic, Newbury, UK). This flow pattern was recorded during a rapid chest compression manoeuvre [1]. Figure 2 illustrates the original flow pattern obtained from an infant and the flows reproduced by the infant lung model. There was generally good agreement between the two signals, except that peak flow was over estimated by up to $10 \%$ by the pump.

Summary. The Squirec linear pump [6] can deliver tidal volumes in the same order of magnitude as observed in infants if two different pistons are used. The accuracy of the pump is good enough to fulfil the ERS-ATS standards if the appropriate piston size is used. The linear pump allows prerecorded flows to be repro- duced as they occur in infants, although, at very large forced peak flows the error was somewhat larger than anticipated from the original description of the pump [6]. Such prerecorded flow patterns may allow infant lung function equipment to be tested with respect to its ability to measure distinct flow points or assessment of triggering mechanisms.

Infant lung model volume measurements. Rationale. In order to measure the volume of a mechanical model $(V \mathrm{~m})$ accurately, both thermal effects during ventilation of the volume elements, as well as pressure losses due to mechanical leaks in the model, must be considered. Theoretically, the faster a fixed mass of gas in a model lung volume is ventilated, the more heat and hence pressure will be generated, which will result in underestimation of the model volume. There are two ideal situations where this phenomenon is precisely calculable. Either no heat is dissipated through the model wall (adiabatic conditions) or all the produced heat is dissipated through the model wall (isothermal conditions) at every pumping frequency. In reality, it is virtually impossible to build an infant lung model that fulfils either of these two extreme conditions, and in most cases the model will behave somewhere between isothermal and adiabatic, this condition being known as polytropic. This polytropic behaviour can be minimized by optimizing the heat exchange through the model wall. The mechanical (leak) time constant will also influence the pressure changes within the model. Leaks can lead to a pressure loss and hence an over estimation of the model volume, especially at lower ventilation rates. All these factors will potentially influence how precisely the model can simulate a given lung model volume. Nevertheless, precision of the model is essential and should be below the recommended measurement variability specified by the recent ERS-ATS standards for infant lung function equipment [2, 3] $(< \pm 2.5 \%$ for infant lung volumes 50-300 mL, minimal $2 \mathrm{~mL}$ ).

Methods. These two potential problems were solved in two ways. Firstly, the model was built so that the
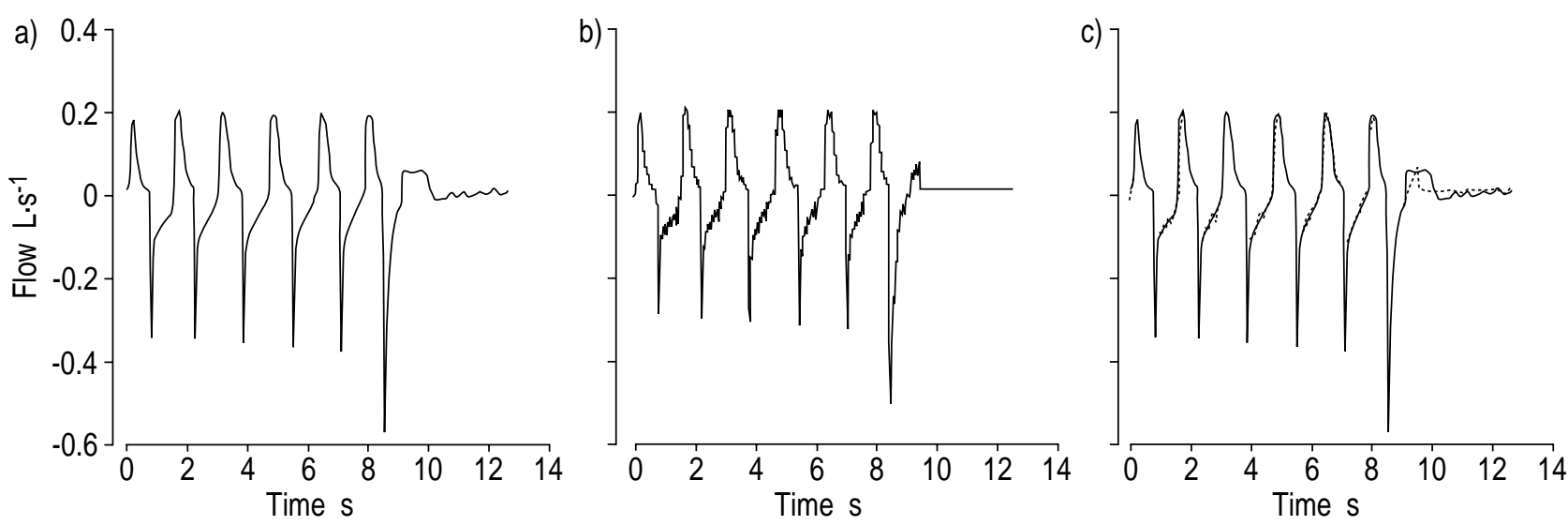

Fig. 2. - Time based recordings of flow during tidal breathing and a forced expiration from a 6 month old infant. a) original recording; b) simulated flow pattern from the pump and; c) overlay of the recorded (solid) and simulated (dotted) flows. Expiration was forced during final breath. 
mechanical leak was small enough and the heat dissipation large enough to ensure reproducible conditions. The model was built of copper and filled with copper wool in order to maximize and homogenize the heat dissipation. The lung model volume elements consisted of copper chambers of approximately 50, $75,100,150$ and $300 \mathrm{~mL}(V 50, V 75, V 100, V 150, V 300$, respectively) which could be connected in series to the linear pump. These $V \mathrm{~m}$ approximate the range of functional residual capacity (FRC) values found in infants $2-12 \mathrm{~kg}$ body weight. The exact volumes of these elements were calculated from water displacement as well as from their physical dimensions, in which both copper wool and connector volumes were taken into account (table 1) [4]. The volume of the copper wool was derived by dividing the weighted mass of the wool by the density of copper. The thermal time constant $\tau$ t for the different lung model volumes was then determined. $\tau \mathrm{t}$ was $0.15 \mathrm{~s}$ for the smallest model volume $(V 50)$ and $0.23 \mathrm{~s}$ for the largest volume $(V 300)$. These time constants indicate that the model was behaving almost, but not entirely, isothermally [1].

The leak of the model (mechanical time constant $(\tau \mathrm{m})$ ) and particularly that of the linear pump, was assessed by applying a step pressure change of 10 $\mathrm{cmH}_{2} \mathrm{O}$ under two conditions: firstly in default position while motionless and secondly during motion while various load capacitances (model lung volumes: V50-300) were connected to the piston. In the motionless condition the time constants of the model were consistently longer than during piston movements. During piston movements with pressure changes $\leqslant 3 \mathrm{kPa}$, $\tau \mathrm{m}$ was $120-150 \mathrm{~s}$ for the smallest model lung volume (V50) and over $500 \mathrm{~s}$ for the largest volume $(V 300)$, indicating that a very small leak was present, the effect of which was more marked at small volumes.

Thus in a second step, the concept of the effective model volume $V$ em was created. This concept is based on the fact that Boyle's law $\left(P_{1} V_{1}=P_{2} V_{2}\right)$ only holds under completely isothermal conditions, but that the errors introduced by thermal effects due to gas compression and pressure losses due to leaks can be measured. In other words, $V \mathrm{em}$ is the volume of the model having taken any over estimation due to gas compression effects and under estimation due to leaks

Table 1. - The exact lung model volumes determined by water displacement

\begin{tabular}{lcccr}
\hline & $\begin{array}{c}V_{\text {water dis }} \\
\mathrm{mL}\end{array}$ & $\begin{array}{c}V_{\text {copper }} \\
\mathrm{mL}\end{array}$ & $\begin{array}{c}V_{\text {calculated }} \\
\mathrm{mL}\end{array}$ & $\begin{array}{r}V_{\mathrm{m}} \\
\mathrm{mL}\end{array}$ \\
\hline Volume 1 & 48 & 1.7 & & 46.3 \\
Volume 2 & 73 & 2.5 & & 70.5 \\
Volume 3 & 98 & 3.4 & & 94.6 \\
Volume 4 & 147 & 5.1 & & 141.9 \\
Volume 5 & 297 & 10.3 & & 286.7 \\
Connector 1 & & & 3.8 & \\
Connector 2 & & & 0.2 & \\
Connector 3 & & & 0.2 & \\
\hline
\end{tabular}

These volumes agreed closely with the volumes calculated from the physical dimensions. The volume effect of the copper wool was calculated from specific weight. into account. Thus the effective $V$ em is that which can be measured under dynamic conditions in an infant whole body plethysmograph, rather than the absolute volume of the model calculated from its physical dimensions.

In order to create calibration curves for effective $V \mathrm{em}$ under various conditions such as pumping frequency and stroke volumes, the copper wool filled copper chambers were connected one at a time in series to the linear pump. The $V \mathrm{~m}$-element was then plugged at the distal end and pressure changes within the $V$ m-element recorded while sinusoidal volume changes of 0.25 $4.2 \mathrm{~mL}$ were applied at frequencies of $10-120 \mathrm{~min}^{-1}$ according to recent specifications [1, 3]. Pressure changes within the model $(P \mathrm{~m})$ were recorded using a piezoresistive pressure transducer (model 33NA-002D; EuroSensor, London, UK; fig. 1). Three sets of 50 tidal pressure changes were measured. Between each set, the set-up was disconnected, reconnected and recalibrated to account for any variability that might occur with different environmental conditions and any error introduced by small variations in the model connectors. From the ratio of the change in pressure $(\Delta P)$ and volume $(\Delta V)$ and the barometric pressure $(P \mathrm{bar})$, the effective $V \mathrm{~m}$ was calculated:

$$
\begin{gathered}
V \text { em }=\frac{[P \text { bar }+\Delta P] \times \Delta V}{\Delta P} \\
\% V \text { em }=\frac{100 \times \text { effective } V \text { em }}{V \mathrm{~m}}
\end{gathered}
$$

These measurements were performed in Berne, Switzerland at a barometric pressure of $96.0 \mathrm{kPa}$, a humidity of $50 \%$ and a temperature of $20^{\circ} \mathrm{C}$. The $V \mathrm{em}$ (percentage of the expected value) of the various $V \mathrm{~m}$ elements was then plotted as a function of ventilatory frequency.

Results. The $\% V$ em (calibration curve) was plotted as a function of breathing frequencies of $10-120 \mathrm{~min}^{-1}$ (fig. 3). Pressure loss due to mechanical leak resulted in a slight overestimation of the model volume particularly at slow breathing frequencies, this effect being most evident when the smallest $V_{\mathrm{m}}(V 50)$ was used (fig. 3a). By contrast, the effects of the polytropic behaviour of the lung model, gas compression effects and gas heating, led to an increase pressure and hence underestimation of gas volume by up to $\sim 10 \%$ at frequencies of $120 \mathrm{~min}^{-1}$. These effects were most relevant when using the larger volume elements (V300 - fig. 3), due to the relatively smaller surface area to volume ratio. Both phenomena in combination lead to a negative frequency dependence of $V \mathrm{em}$. Nevertheless, from the calibration curves in figure 3, every expected $V$ em can be derived under various conditions [4]. For all lung model elements, the 95\% confidence intervals for measured volumes were $< \pm 1 \%$ of the corresponding mean at every frequency and tidal volume. 

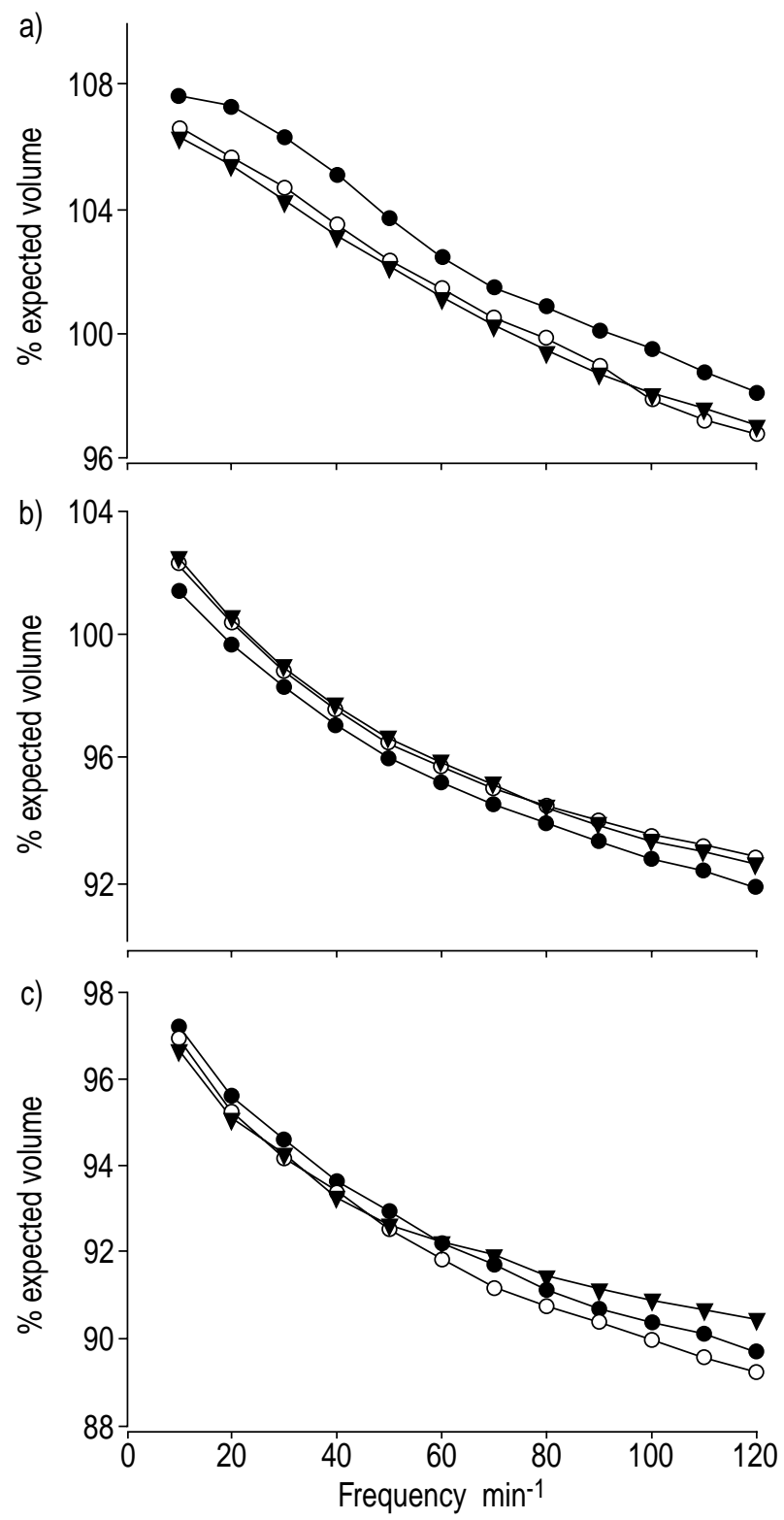

Fig. 3. - Shows the effective volume of the model lung $\left(V_{\mathrm{em}}\right)$ as a function of frequency for model volumes (V50-300). a) $50 \mathrm{~mL}$ volume $\left(V_{50}\right) ; 0$ : $\left.V_{\mathrm{T}}=0.25 \mathrm{~mL} ; \bigcirc: 0.5 \mathrm{~mL} ; \boldsymbol{\nabla}: V_{\mathrm{T}}=1 \mathrm{~mL} . \mathrm{b}\right)$ $100 \mathrm{~mL}$ volume $\left(V_{100}\right) ; 0: V \mathrm{~T}=0.5 \mathrm{~mL} ; \bigcirc: V_{\mathrm{T}}=1.5 \mathrm{~mL} ; \boldsymbol{\nabla}$ $V_{\mathrm{T}}=2.5 \mathrm{~mL}$. c) $300 \mathrm{~mL}$ volume $\left(V_{300}\right) ; 0: V_{\mathrm{T}}=2.5 \mathrm{~mL} ; \bigcirc$ $V \mathrm{~T}=3.5 \mathrm{~mL} ; \boldsymbol{\nabla}: V_{\mathrm{T}}=4.2 \mathrm{~mL}$. Due to a small mechanical leak of the lung model, $V$ em was overestimated by $\sim 5 \%$ for small lung volumes $\left(V_{50}\right)$ at very low frequencies $\left(<10 \cdot \mathrm{min}^{-1}\right)$. This was less of a problem for large volumes $(V 300)$ where capacitance was larger and $\tau_{\mathrm{m}}$ therefore longer. By contrast, thermal effects due to gas compression heating are more relevant for large volumes since relative surface area is smaller. Thus for large volumes ( $V 300$ ) volume is underestimated $\sim 5 \%$ at a frequency of $30 \mathrm{bpm}$.

Summary. By creating a series of calibration curves, it was possible to account for the physical limitations of the model with respect to the underlying assumptions of Boyle's law. The calibration curve in figure 3 can be used to predict the model volume, $V \mathrm{~m}$, that an infant whole body plethysmograph should be able to measure in a test situation. The precision $( \pm 1 \%)$ with which the infant lung model could rep- roduce effective volumes at any given frequency and stroke volume was below the required measurement variability $(< \pm 2.5 \%)$ for volumes $50-300 \mathrm{~mL}$ and pumping frequencies of $20-120 \mathrm{~min}^{-1}$.

Resistance measurements. Rationale. Infants have relatively high airway resistance [7]. It is difficult to construct physical flow resistors in the same order of magnitude since such high resistors usually show highly nonlinear behaviour. Turbulent flows, which may easily occur at the flows observed in infants $\left( \pm 0-0.3 \mathrm{~L} \cdot \mathrm{s}^{-1}\right)$, cause a large variability in resistance. These factors potentially influence how precisely any model can simulate a given flow resistor. Nevertheless, precision of the model is essential and should be below the required measurement variability specified for infant lung function equipment by the recent ERS-ATS standards $[2,3]$ (i.e. $< \pm 5 \%$ for infant flow resistances $0.5-10 \mathrm{kPa} \cdot \mathrm{L}^{-1} \cdot \mathrm{s}$, minimal $\left.0.01 \mathrm{kPa} \cdot \mathrm{L}^{-1} \cdot \mathrm{s}\right)$.

A second problem has to be solved if model flow resistors are to be used in plethysmographs. If the pump was directly connected to the flow resistor, there would be instantaneous equilibration and hence no pressure change in the plethysmograph. In order to achieve significant pressure changes in the plethysmograph, a model volume (acting as a capacitance) has to be connected between the flow resistor and the model pump. However, such a volume can potentially influence the pressure/flow relationship, introducing a phase lag and hence causing looping of the pressure flow curve, particularly at higher pumping frequencies. Thus, the optimal setup has to be found, which on the one hand allows accurate resistance measurements in the absence of any phase lag, but on the other hand allows significant pressure changes within the plethysmograph.

Methods. To simulate airway resistance in the infant model, screen resistors were constructed from sinter material (Meyer Sintermetall AG, Studen, Switzerland). This sinter material was made of bronze metal powders, lumped together to form screens. The diameter varied $22-30 \mathrm{~mm}$ and depth varied $0.8-4.3 \mathrm{~mm}$ [4]. Dependent on particle sizes, the resistive properties varied. Small particles resulted in high resistance and large particles in low resistance. Five different resistor screens $(R 1-5)$ were built (table 2$)$ exhibiting

Table 2. - Resistive properties of the resistor screens $(R 1-5)$ derived from linear regression

\begin{tabular}{lcc}
\hline Resistance & Flow range $\pm 0.1 \mathrm{~L} \cdot \mathrm{s}^{-1}$ & Flow range $\pm 0.3 \mathrm{~L} \cdot \mathrm{s}^{-1}$ \\
\hline$R 1$ & $0.75(0.71-0.79)$ & $1.01(0.98-1.04)$ \\
$R 2$ & $0.89(0.84-0.94)$ & $1.32(1.23-1.41)$ \\
$R 3$ & $1.08(1.03-1.13)$ & $1.47(1.44-1.50)$ \\
$R 4$ & $2.12(2.00-2.24)$ & $2.88(2.74-3.02)$ \\
$R 5^{*}$ & $3.93(3.81-4.05)$ & $5.57(5.21-5.93)$ \\
\hline
\end{tabular}

Slopes of linear regression (95\% confidence interval) are shown over two different flow ranges, namely $-0.1-0.1 \mathrm{~L} \cdot \mathrm{s}^{-1}$ and $-0.3-0.3 \mathrm{~L} \cdot \mathrm{s}^{-1}$ respectively. *: the screen $R 5$ showed sufficient reproducibility at a flow range of $-0.1-0.1 \mathrm{~L} \cdot \mathrm{s}^{-1}$ but was more variable from $-0.3-0.3 \mathrm{~L} \cdot \mathrm{s}^{-1}$. The correlation coefficient $\left(\mathrm{r}^{2}\right)$ was $>0.99$ in all measurements. 


\section{Exchangeable}

resistance

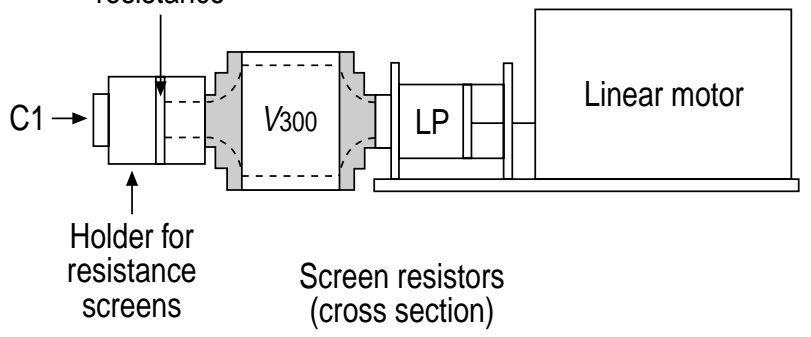

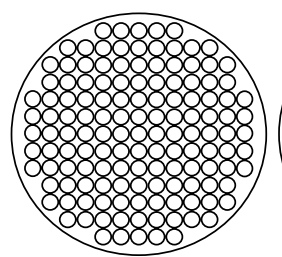

R2

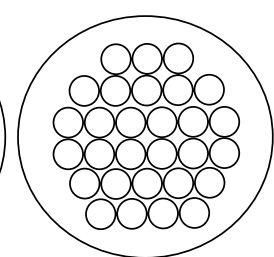

$R 3$

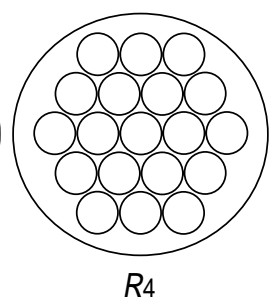

R4
Fig. 4. - Diagram of lung model attached to various flow resistors. $\mathrm{C} 1$ connected the set-up to the pneumotachometer; $R 2-4$ : resistors $2-4$. $\square$ : copper adaptors.

airflow resistances $1-5.6 \mathrm{kPa} \cdot \mathrm{L}^{-1} \cdot \mathrm{s}$ at flows $0-0.3 \mathrm{~L} \cdot \mathrm{s}^{-1}$. These screen resistor elements could be connected in series to the capacitative volume element (V300) and then to the model pump (fig. 4).

With these optimized flow resistors, a similar approach as described previously in "infant lung model volume measurements" was used. Calibration curves were created for various flow resistors under various conditions such as changing flows, so that when testing infant lung function equipment, the simulated airflow resistance could be derived from these calibration curves. To test the physical properties of the various resistor screens and to determine these calibration curves, a tidal flow of $-0.3-0.3 \mathrm{~L} \cdot \mathrm{s}^{-1}$ was applied and the pressure drop across the resistor was measured using a piezoresistive pressure transducer (EuroSensor 33NA-002D). Flow was monitored using a pneumotachograph (model PT36; Erich Jaeger GmbH, Hoechberg, Germany; frequency response: flat up to $100 \mathrm{~Hz}$ ) and a Honeywell pressure transducer (model 163 PC, range $\pm 6.23 \mathrm{cmH}_{2} \mathrm{O}$; Honeywell, Freeport, IL, USA). Three sets of 20 tidal pressure/flow changes were measured. Between each series, the set-up was disconnected, reconnected and recalibrated to account for any variability that might occur with different environmental conditions or errors introduced by small variations in the model connectors. The pressure flow relationship was fitted using both linear regression over the flow range $-0.1-0.1 \mathrm{~L} \cdot \mathrm{s}^{-1}$, and $-0.3-0.3 \mathrm{~L} \cdot \mathrm{s}^{-1}$ and by fitting third order polynomial functions. In order to find the optimal set-up of the model and its optimal capacitance, the phase relationship between pressure and flow was tested while model volumes of different sizes were connected $(V 50-300)$ between the flow resistor and the model pump.

Results. The use of $V 300$ allowed significant box volume changes in the absence of looping up to $2 \mathrm{~Hz}$ [5], whereas volumes $<200 \mathrm{~mL}$ did not, which would

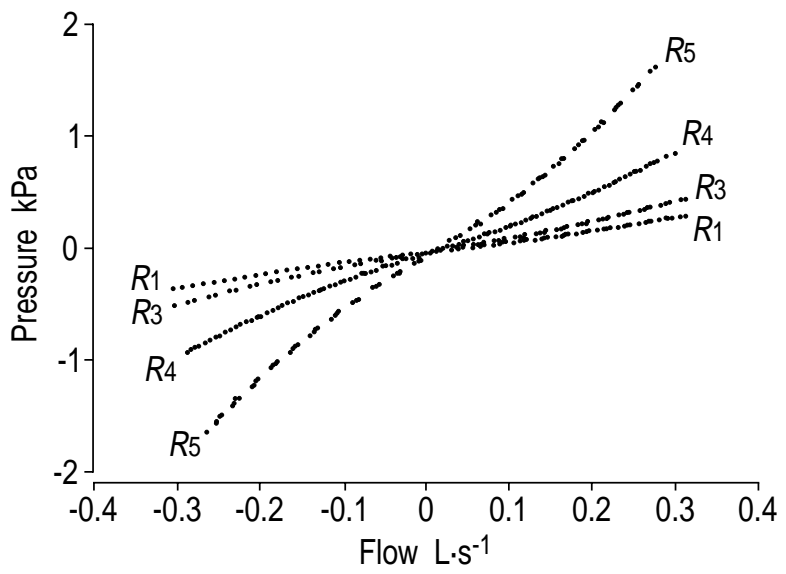

Fig. 5. - Pressure/flow relationship across the various screen resistor elements $(R 1,3,4,5) . R 2$ is not shown for graphical reasons, but lays between $R 1$ and $R 3$. The flow range represents that commonly observed during tidal breathing in infants $<10 \mathrm{~kg}$. The resistors used show differences in curvatures among pressure flow relationship.

have caused signal to noise problems. Thus, the calibration curves for the different resistor elements were determined using the model volume $V 300$ (fig. 5). The resistance of the various elements, together with their $95 \%$ confidence intervals, was assessed from three sets of 20 measurements and are presented in table 2 . Linear regression is a practical simplification that is particularly useful in the tidal flow range. However, the whole pressure/flow relationship can more adequately be expressed by a cubic polynomial function of the following form (table 3):

$$
P=\mathrm{k}+\mathrm{a}\left(V^{\prime}\right)+\mathrm{b}\left(V^{\prime}\right)^{2}+\mathrm{c}\left(V^{\prime}\right)^{3}
$$

Summary. Flow resistors made of pressed metal powder provided airflow resistance values up to 5.6 $\mathrm{kPa} \cdot \mathrm{L}^{-1} \cdot \mathrm{s}$ at flows ranging $-0.3-0.3 \mathrm{~L} \cdot \mathrm{s}^{-1}$. Such flow resistors are able to simulate airway resistance in infants. The precision of the resistance simulations was found to be $< \pm 2.5 \%$ of the mean, which is within the limits suggested by the recent ERS-ATS standards [3]. There was no significant looping between pressure

Table 3. - Resistive properties of the resistor screens $(R 1-5)$ derived from polynomial fitting of the pressure/flow relationship

\begin{tabular}{llllc}
\hline Screens & \multicolumn{1}{c}{$\mathrm{k}$} & \multicolumn{1}{c}{$\mathrm{a}$} & \multicolumn{1}{c}{$\mathrm{b}$} & $\mathrm{c}$ \\
\hline$R 1$ & -0.0370 & 0.8990 & -0.0205 & 1.88 \\
$R 2$ & -0.0211 & 1.07 & -0.121 & 3.81 \\
$R 3$ & -0.0373 & 1.27 & -0.0427 & 3.23 \\
$R 4$ & -0.046 & 2.48 & -0.157 & 6.91 \\
$R 5$ & -0.0712 & 4.7 & 0.231 & 19.3 \\
\hline
\end{tabular}

Pressure/flow relationship of the screen resistors over the entire flow range using a third order polynomial function of the form: $P=\mathrm{k}+\mathrm{a}\left(V^{\prime}\right)+\mathrm{b}\left(V^{\prime}\right)^{2}+\mathrm{c}\left(V^{\prime}\right)^{3}$. Pressure is represented in $\mathrm{kPa}$ and flow in $\mathrm{L} \cdot \mathrm{s}^{-1}$. The correlation coefficient $\left(\mathrm{r}^{2}\right)$ was $>0.99$ in all measurements. 
and flow at frequencies up to $120 \mathrm{~min}^{-1}$, even when a model volume of $V 300$ was connected between the model pump and the screen resistor. This optimal setup allows significant pressure changes within an infant plethysmograph with a negligible signal to noise ratio [5].

Assessment of the frequency response functions of the infant lung model. Rationale. Infant lung function equipment needs to be tested regarding its frequency response [2, 3]. This is particularly important if measurements are dependent on $\geqslant 2$ signals. During plethysmographic resistance measurements, a phase lag between box pressure and airflow at the mouth can either be caused by lung function abnormalities or by physical properties of the lung function equipment. Thus, it is important to ascertain that the equipment itself does not introduce a phase lag or looping between signals. For such measurements the ERS-ATS standards specify fidelity of the equipment of $\pm 10 \%$ in magnitude and $\pm 3^{\circ}$ in phase up to $10 \mathrm{~Hz}$ $[2,3]$. In most cases this can be assessed traditionally using loudspeakers [8-11], but under specialized conditions such as in infant plethysmographs, where signals of very different magnitude (box pressure ( $P$ box $)$, airway opening pressure $(P$ ao $)$, flow) are to be compared and signal transducers are built into the equipment, an infant lung model is advantageous [5].

Methods and results. As previously described, the Scireq linear pump can deliver small amplitude sinusoidal volumes up to $20 \mathrm{~Hz}$ [6], thereby allowing the assessment of frequency response of equipment. However, if the pump is connected to an infant lung model, the physical properties of this model may influence pressure and flow changes measured at the outlet of the model (position $P \mathrm{~m}$ ). Thus, the frequency response function of the infant lung model itself has to be determined before using it to assess that of infant lung function equipment.

To do this, the amplitude and phase relationship between the oscillatory piston volume signal, $V \mathrm{p}(\omega)$, and the resulting oscillatory pressure changes, $\operatorname{Pm}(\omega)$, was determined while the model was closed at the proximal end (position $P \mathrm{~m}$ ). This set-up simulates plethysmographic lung volume measurements whereby $P$ m represents $P$ ao. The pump was connected to a known volume $(V 150)$ and then used to generate a pseudorandom noise signal $(V \mathrm{p}(\omega))$ ranging $0.25-12 \mathrm{~Hz}$. The resulting oscillatory pressure changes were measured at the pressure port near the model outlet $(P \mathrm{~m}(\omega))$ using a solid state piezoresistive pressure transducer (EuroSensor 33NA-002D). $P \mathrm{~m}(\omega)$ and $V \mathrm{p}(\omega)$ were AD-converted with a sampling rate of $256 \mathrm{~Hz}$ and an accuracy of 12 bit. The phase relationship induced by the physical characteristics of the model was calculated from the complex ratio $V \mathrm{p}(\omega) /$ $P \mathrm{~m}(\omega)$. Figure 6 shows the normalized magnitude for $V \mathrm{p}(\omega)$ and the phase of the frequency response function for $V \mathrm{p}(\omega) / P \mathrm{~m}(\omega)$. There was no significant change of magnitude up to $12 \mathrm{~Hz}$, whereas there was a frequency dependent increase in phase shift between $V \mathrm{p}(\omega)$ and $P \mathrm{~m}(\omega)$. The latter was mainly due to the capacitative and thermal properties of the gas in the model volume.
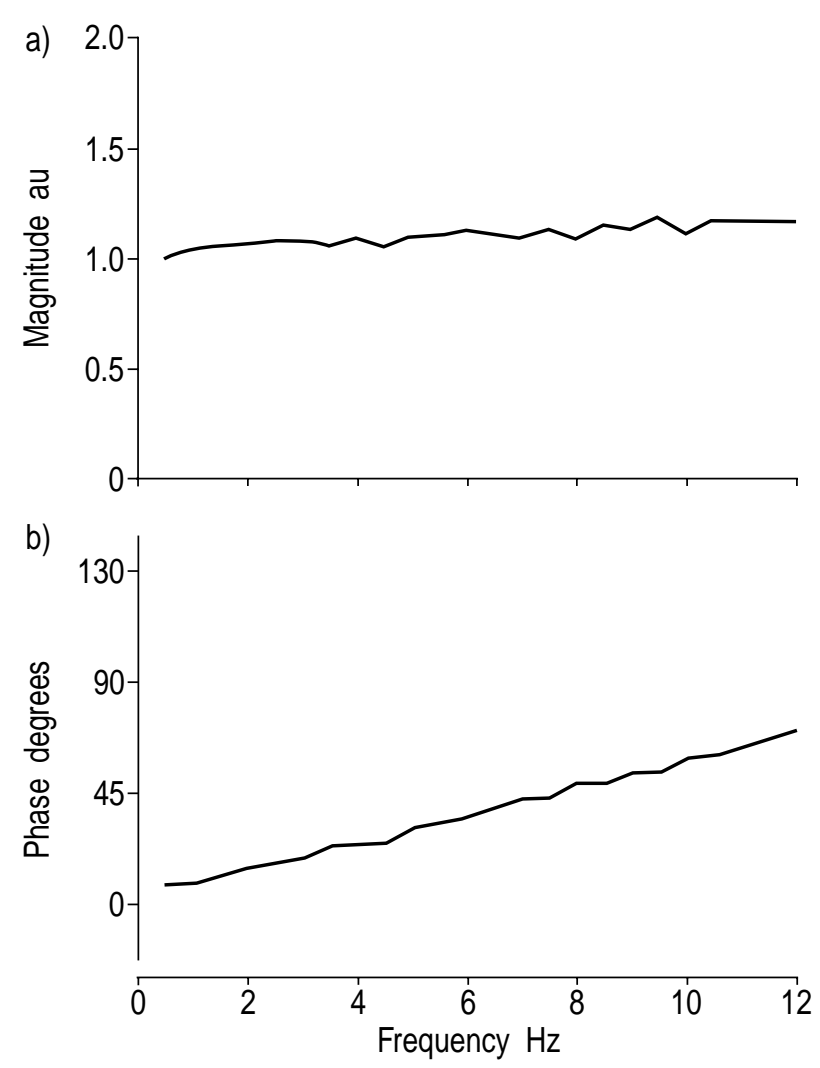

Fig. 6. - Amplitude/normalized magnitude (a) and phase relationship (b) between piston volume $V_{\mathrm{p}}(\omega)$ and airway opening pressure $P \mathrm{~m}(\omega)$ in the infant lung model when the proximal end (position of $P \mathrm{~m}$ on fig. 1) is closed.

Similarly, the frequency response function between $V \mathrm{p}(\omega)$ and the resulting oscillatory flow changes $\left(V^{\prime}(\omega)\right)$ was determined when the model was connected to the resistor element at its proximal end. In this set-up, the pump was connected to $V 300$, since signal to noise ratio was better using a larger model volume. This set-up simulates plethysmographic resistance measurements. $V^{\prime}(\omega)$ was measured using a PT36 pneumotachometer (model $163 \mathrm{PC}$, range $\pm 6.23 \mathrm{cmH}_{2} \mathrm{O}$; Honeywell) at the model outlet port. The signals were sampled and the frequency response function between $V \mathrm{p}(\omega)$ and $V^{\prime}(\omega)$ was calculated accordingly. Figure 7 shows the normalized magnitude for $V \mathrm{p}(\omega)$ and the phase of the frequency response function of $V \mathrm{p}(\omega) / V^{\prime}(\omega)$. The frequency dependent increase in magnitude and decrease in phase shift between $V \mathrm{p}(\omega)$ and $V^{\prime}(\omega)$ was mainly due to the decrease of $V^{\prime}(\omega)$, because of the capacitative properties of the gas in the model volume.

Summary. The frequency response characteristics of the infant lung function model was determined while stimulating both lung volume and airway resistance measurements. Once these functions had been determined, they could be compensated for when using the model to assess the frequency response of equipment such as infant plethysmographs [5]. 

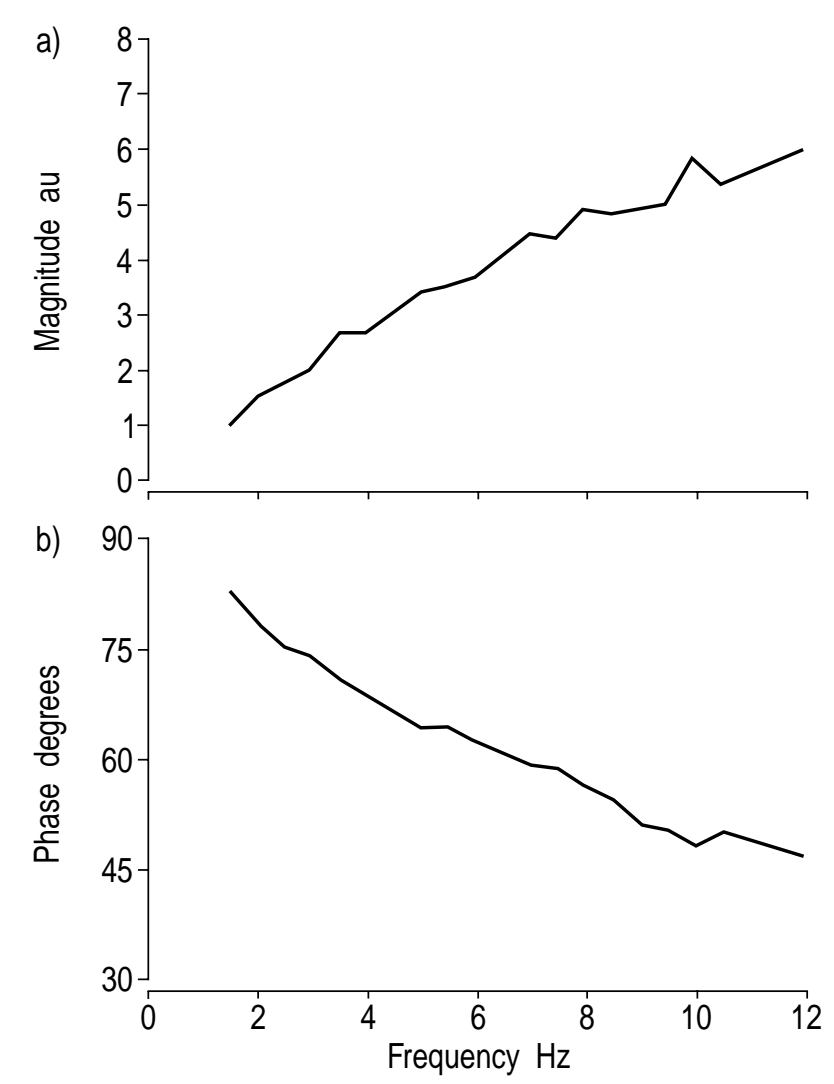

Fig. 7. - Amplitude/normalized magnitude (a) and phase relationship (b) between piston volume $V \mathrm{p}(\omega)$ and flow $V^{\prime}(\omega)$ in the infant lung model when the proximal end (position of $P \mathrm{~m}$ ) in open.

\section{Discussion}

The results from this study demonstrate that it is possible to develop a mechanical infant lung function model that can reliably reproduce flow patterns of infants, and simulate lung volumes and airflow resistors. The model is a simple one compartment system and does not simulate multiple compartments of the infant's lung or any humidity or warming effects introduced by the infant's body temperature. The precision of the infant lung model was sufficiently good to allow it to be used to assess whether infant lung function equipment meets ERS standards [3] (volumes $\pm 5 \%$, flows $\pm 2.5 \%$, resistances $\pm 5 \%$, frequency response up to $10 \mathrm{~Hz}$ ). However, this was an extremely demanding undertaking, since the precision of the test device is in turn influenced by various factors such as nonlinear flows due to turbulence, polytropic thermal effects, leak and the mechanical behaviour of moving parts (e.g. piston) of the model. The validity, potential confounding factors and implications of the model are now discussed.

The dimensions of the infant lung model were small enough for it to be used even within an infant whole body plethysmograph [5]. The linear pump did not produce any vibration or heat [5] which allowed assessment of infant lung function equipment with a good signal to noise ratio. Nevertheless, the advantages of a linear pump are traded against the disadvantage of the motor being only just powerful enough to drive the piston, and the fact that the piston seal cannot be made entirely leak free. The time constants of the model were however, long enough to be reproducible and mathematically calculable (see later).

\section{Tidal volume and flow measurements}

The design of the pump and model were selected to ensure that they would function over the range of flow, resistance and volume changes normally encountered during infant lung function tests. Tidal flows in infants up to $10 \mathrm{~kg}$ range $0-0.3 \mathrm{~L} \cdot \mathrm{s}^{-1}$, but increase to 0.6 or $1.5 \mathrm{~L} \cdot \mathrm{s}^{-1}$, respectively, if partial or raised lung volume forced expiratory manoeuvres are generated $[1,3,12]$. The model described in this paper can reproduce given flows from $0-1.5 \mathrm{~L} \cdot \mathrm{s}^{-1}$.

The precision at which flows and volumes can be delivered by the linear pump has been described previously as being $0.5 \%$ of the full stroke [6]. However, if only $10 \%$ of the full stroke volume is delivered, this error can rise to $5 \%$ of the delivered volume. Thus in order to optimize the tidal volume precision, the piston has to be chosen so that the delivered volume is close to full stroke volume. Similarly, when using the model pump to simulate prerecorded flow patterns, an optimal piston with a full stroke volume close to that of the simulated volume signal should be used to minimize the error. During the current study, it was found that the error was particularly large at points of rapid flow changes or turning points (e.g. peak flows) possibly due to the inertia of the piston and a small mechanical variability of the pump. Nevertheless, the ability of the pump to simulate prerecorded flows will be useful for generating standardized flow patterns from infants of different age and clinical status. Using such simulations, the accuracy with which lung function equipment can analyse both tidal and forced expiratory flow patterns, or trigger valves from tidal flow or volume signals, could be tested prior to releasing equipment for use in infants. In the future, establishment of a database containing standard infant breathing patterns, that could be used by both clinicians and manufacturers to test equipment, would be extremely useful.

\section{Model volume measurements}

By creating calibration curves for the effective lung model volumes, it was possible to account for the small pressure changes in the model due to both mechanical leaks and the polytropic behaviour of the infant lung model. Pressure losses due to mechanical leak resulted in an overestimation of the model volume, particularly at slow pumping frequencies in small volumes (fig. 3). On the other hand, gas compression and heating effects led to an increase in recorded pressure changes and hence underestimation of model volume, particularly at high frequencies in larger volumes (fig. 3). Both phenomena in combination, led to a negative frequency dependence of $V \mathrm{em}$. Nevertheless, by using the calibration curves shown in figure 3 , the 
model can be used to assess how accurately an infant whole body plethysmograph can assess lung volumes in vitro. The precision and reproducibility of the infant lung model in measuring $V$ em at any given frequency and stroke volume was $< \pm 1 \%$, while the required measurement variability was $< \pm 2.5 \%$ at volumes ranging 50-300 mL, over a wide range of frequencies, well within the recommended limits [2, 3]. It should be noted however, that since the effective volumes presented in figure 3 were measured at a $P$ bar of $96.0 \mathrm{kPa}$, the values would need to be corrected to the current barometric pressure by a simple ratio if they were to be used in different laboratories. Thus, if measurements were to be performed at sea level, a correction of around $6.6 \%$ would be required.

It should be noted that thermal effects and variability of stroke volume only play a role if the volume of the model is measured indirectly, by methods such as plethysmography. By contrast, if the volume of the model were to be measured using a gas dilution technique, then the leak problem becomes more complex. The leak time constant of any model will then depend not only on the motion of the piston, but also on gas density, particularly if a highly diffuse gas is used. In this study, the leak behaviour in the presence of gases other than air was not calculated. However, since the lung model volume can be sealed completely when disconnected from the pump it could also be used to assess gas washout techniques by using airtight syringes. In such circumstances thermal effects within the model and the syringe would be less critical. The model volumes (V50-300) would then correspond to the physical volumes (including connectors) determined by water displacement (table 1).

\section{Model resistance measurements}

The infant lung model is capable of reproducing resistances of $1-5.6 \mathrm{kPa} \cdot \mathrm{L}^{-1} \cdot \mathrm{s}$, which equates to the range of airway resistances normally encountered in infants up to $10 \mathrm{~kg}[1,3,7]$. Before finally selecting pressed metal powder screens, attempts were made to use various materials, such as bundles of thin tubes, containers of small glass spheres, screen resistors, fibre materials and coned tubes, in order to simulate such high flow resistances. All of these systems showed a highly nonpredictable, nonlinear flow behaviour, or did not constitute a high enough resistance. By contrast, resistors made of pressed metal powder showed approximately linear pressure/flow relationships with excellent reproducibility $( \pm 2.5 \%)$ at flows $-0.1-$ $0.1 \mathrm{~L} \cdot \mathrm{s}^{-1}$. The pressure flow relationships at flows ranging $-0.3-0.3 \mathrm{~L} \cdot \mathrm{s}^{-1}$ could be fitted exactly using polynomials of the third order, whereas the traditional second order Rohrer's equations did not fit these adequately. The regression parameters $\mathrm{a}, \mathrm{b}$ and $\mathrm{c}$ (table 3) cannot be related to a distinct physical property of the system. However, for simplicity and practical use, linear regression (correlation coefficient $>0.99$ ) can be used, particularly over the flow range $-0.1-0.1 \mathrm{~L} \cdot \mathrm{s}^{-1}$. Since the model was capable of simulating flow resistances with a precision of $\pm 2.5 \%$, it can be used to assess whether infant lung function equipment is able to measure such resistances with an accuracy of $\pm 5 \%$. Of greater relevance was the fact that the model itself was capable of simulating resistances within the range normally found during infant respiratory function tests [3]. These model resistor screens could also be used to assess airflow resistance by other techniques, such as the interrupter technique or the forced oscillation technique.

Assessment of the frequency response functions of the infant lung model

Traditionally, loudspeakers can be used to test the frequency response of equipment [8-11]. However, under certain conditions, such as during the testing of the frequency response of an infant whole body plethysmograph, it is not possible to expose two or more transducers directly to the same oscillatory pressure changes. This is because even small oscillatory pressure changes could drive the box pressure transducer to the limits of its physical pressure range, whereas such oscillations when measured by the mouth pressure transducer, which has a much wider pressure range, would hardly be distinguishable from environmental noise. Thus, the amplitudes of the oscillatory pressure changes had to be adjusted so that both pressure transducers were well within their optimal working range. This was easily achieved by connecting the infant lung model between the pump (oscillator) and the $P \mathrm{~m}$ transducer. The volume in the model then acted as a capacitance to scale the pressure swings down to a magnitude that was within the range of transducers used to measure $P$ ao in an infant plethysmograph. However, this advantage was traded against the disadvantage that the infant lung model itself introduces some frequency dependent phase lag between these two signals, because it acts mainly as gas compression compliance. Nevertheless, having established the frequency response functions of the infant lung model, both when the model was closed at its outlet (representing the plethysmographic FRC measurements) and when it was open (representing the plethysmographic airway resistance (Raw) measurements), these can be taken into account mathematically when testing infant lung function equipment.

An infant lung function model has been developed which meets the criteria recently recommended by the European Respiratory Society-American Thoracic Society (ERS-ATS) task force for infants [3]. This model can be used not only to assess the accuracy of either plethysmographic or gas dilution techniques in measuring lung volumes, but can also be used to simulate plethysmographic assessments of airway resistance. Since the model has the ability to reproduce sinusoidal flows at frequencies up to $10 \mathrm{~Hz}$, it is also a useful means of testing the phase relationship between any two measurement signals. This is crucially important in plethysmography, forced oscillation techniques (pressure and flow) and in pulmonary resistance measurements (oesophageal pressure versus airflow). Finally, one of the major advantages of the model is its ability to reproduce prerecorded flow patterns [6]. Thus, the ability of lung function equipment 
to measure tidal or forced flow patterns accurately can be assessed using standardized prerecorded flow patterns. Potentially, this could be combined with volume and resistor elements respectively, so that complex situations could be simulated. Furthermore, isolated use of some of the individual components could extend the applications of this model. For example, if only plethysmographic volumes were of interest, a simple sinusoidal pump could be connected to the model volume elements. Manufacturers could build such simplified models to facilitate regular checking of their equipment and to allow rapid comparison of systems between laboratories. It should however be noted, that before the latter can be achieved, standardized testing protocols need to be established. The latter was beyond the scope of this current technical paper, but presents a worthwhile challenge for the future. Nevertheless, this infant lung model could serve as a prototype for a future generation of "breathing mechanical model babies".

Acknowledgements. The authors thank $\mathrm{T}$. Schuessler and J.H.T. Bates for their technical support.

\section{References}

1. Stocks J, Sly PD, Tepper RS, Morgan WJ, eds. Infant respiratory function testing. New York, Wiley-Lyss, 1996.

2. Frey U, Stocks J, Sly P, Bates JHT. Specifications for signal processing and data handling used in infant pulmonary function testing. Eur Respir $J$ 2000; 16 : $1016-1022$

3. Frey U, Stocks J, Coates A, Sly P, Bates JHT. Specifications for equipment used for infant pulmonary function testing. Eur Respir J 2000; 16: 731-740.

4. Reinmann B. The infant lung function model, a mechanical analog to test infant lung function equipment. MD thesis 1999, University of Berne, Switzerland.

5. Reinmann B, Stocks J, Frey U. Assessment of an infant whole-body plethysmograph using an infant lung function model. Eur Respir J 2000; 17: 765-772.

6. Schuessler TF, Bates JHT. A computer-controlled research ventilator for small animals: Design and evaluation. IEEE Trans Biomed Eng 1995; 42: 860-866.

7. Stocks J, Godfrey S. Specific airway conductance in relation to postconceptional age during infancy. $J$ Appl Physiol 1977; 43: 144-154.

8. Jackson AC, Vinegar A. A technique for measuring frequency response of pressure, volume and flow transducers. J Appl Physiol 1979; 47: 462-467.

9. Jackson AC. Dynamic response of transducers used in respiratory physiology Part II. In: Otis $\mathrm{AB}$, ed. Techniques in the Life Sciences. Techniques in Respiratory Physiology, Vol P4/II. Gainesville, FL; Elsevier Scientific, 1984; pp. 411-418.

10. Proulx PA, Harf A, Lorino H, Atlan D, Laurent D. Dynamic characteristics of air-filled differential pressure transducers. J Appl Physiol 1979; 46: 608-614.

11. Vallinis P, Retvalvi S, Davies GM, Coates AL. A simplified method for determining the frequency response of pneumotachographs used in infants. Pediatr Pulmonol 1993; 16: 109-115.

12. Sly P, Tepper R, Henschen M, Gappa M, Stocks J. Tidal forced expirations. Eur Respir J 2000; 16: 741748. 